\title{
Mitigation of thermal distortion during additive manufacturing
}

\author{
T. Mukherjee ${ }^{1}$, V. Manvatkar ${ }^{1}, A$. De $^{1}$ and T. DebRoy ${ }^{1}$ \\ ${ }^{1}$ Department of Materials Science and Engineering \\ The Pennsylvania State University, University Park, PA, 16801, USA
}

\begin{abstract}
Additively manufactured parts are often distorted because of spatially variable heating and cooling. Currently there is no practical way to select process variables based on scientific principles to alleviate distortion. Here we develop a roadmap to mitigate distortion during additive manufacturing using a strain parameter and a well-tested, three-dimensional, numerical heat transfer and fluid flow model. The computed results uncover the effects of both the key process variables such as power, scanning speed, and important non-dimensional parameters such as Marangoni and Fourier numbers and non-dimensional peak temperature on thermal strain. Recommendations are provided to mitigate distortion based on the results.
\end{abstract}

Keywords: Additive manufacturing, 3D printing, Laser deposition, Thermal strain

Laser assisted additive manufacturing (AM) process produces 'near net shape' parts from a stream of alloy powders in a layer-by-layer manner for use in medical, aerospace, automotive and other industries [1,2]. The parts undergo repeated spatially variable heating, melting, solidification and cooling during AM [3,4]. Due to the transient heating and cooling, the fabricated parts exhibit thermal distortion [5-10]. Thermal distortion results in dimensional inaccuracy and adversely affects performance of the fabricated parts [11]. Previous work has shown that increase in net heat input [7] and reduction in dwell time [8] between deposition of successive layers can increase thermal distortion. It is also known that the alloy properties, the deposit and substrate dimensions, the laser scanning pathway, the hatch spacing between layers and the heating and cooling conditions significantly affect thermal distortion $[9,12,13]$.

The existing AM literature does not provide any guidance for selecting process variables to minimize thermal distortion. A quantitative understanding of the effects of process variables on thermal distortion and a practical means to mitigate this problem based on scientific principles are needed but not generally available.

Here, we show for the first time how thermal distortion during AM can be minimized by back of the envelope calculations. The procedure involves evaluation of the effects of common 
process variables such as laser power and scanning speed on thermal strain. In addition, the nondimensional parameters that are important for heat transfer and fluid flow phenomena in AM such as Fourier number, Marangoni number and non-dimensional temperature are correlated with thermal strain. Based on these results we provide recommendations to minimize distortion of the additively manufactured parts.

We have recently shown that thermal distortion is related to a strain parameter, $\varepsilon^{*}[5]$ :

$$
\varepsilon^{*}=\frac{\beta \Delta T}{E I} \frac{t H^{3 / 2}}{F \sqrt{\rho}}
$$

where $\beta$ is the volumetric coefficient of thermal expansion, $\Delta T$ is the maximum rise in temperature during the process, $E$ is the elastic modulus and $I$ is the moment of inertia of the substrate, the product, $E I$, is the flexural rigidity of the structure, $\mathrm{t}$ is the characteristic time, $H$ is the heat input per unit length, $F$ is the Fourier number and $\rho$ is the density of the alloy powder. Fourier number is the ratio of the heat dissipation rate to heat storage rate. The temperature rise and the Fourier number are both calculated using a well-tested heat transfer and fluid flow model $[3,4]$. The model solves the equations of conservations of mass, momentum and energy to provide three dimensional transient temperature and velocity fields as well as the shape and size of the molten pool $[3,4]$. Since the model and its applications are described in detail in the literature [3-5], they are not repeated here.

Figure 1 shows the computed temperature and velocity fields, and the shape and size of molten pools for three alloys during the deposition of the second layer. The thermo-physical properties of the alloys [14] used for the calculations are presented in Table 1. Each color band in Figure 1 represents a temperature range as shown in the legend. The melt pool is indicated by the red colored region where the temperature is above the liquidus temperature of the alloy. Likewise, the region where the temperature reaches between the solidus and liquidus temperature is indicated by the green color. The computed velocity fields result from the spatial gradient of surface tension on the surface of the molten pool, also known as the Marangoni stress. A reference vector is shown by an arrow and a comparison of the length of this arrow with the vectors in the plots indicates the magnitudes of the computed velocities. Figure 1 shows that the melt pool size of Ti-6Al-4V is the largest among the three alloys mainly because of its lowest density compared to those of the other alloys as shown in Table 1. 
Equation (1) does not consider any plastic deformation of the structure. However, the causative factors for plastic deformation are the same as those for the strain parameter, $\varepsilon^{*}$, expressed by equation (1). A quantitative relation between the maximum thermal strain encountered during deposition and the strain parameter $\varepsilon^{*}$, as shown in Figure 2, was established in a recent paper [5]. It is observed that the maximum measured strain in multi-layer deposition correlates linearly with the strain parameter, $\varepsilon^{*}$, for several alloys and various processing conditions [5]. Thus, the strain parameter, $\varepsilon^{*}$, is considered to be a useful indicator of thermal distortion for the deposition of various alloys. This parameter embodies all causative factors for thermal distortion but, like all scaling analysis, does not provide an exact magnitude of strain. Valuable insight, not precision, is what the strain parameter provides. The measured strain is not zero at very low values of strain parameter because non-dimensional analysis is not meant to give the exact value. The strain parameter is important because it provides for the first time a clear understanding of how the strain will change with various factors as shown by equation (1).

Here we examine the non-dimensional thermal strain for the depositions of SS 316, Ti6Al-4V and IN 718 alloys using equation (1) and parameters computed from a heat transfer and fluid flow model. The calculations were done for laser powers and scanning speeds in the commonly used ranges of 190 to $270 \mathrm{~W}$ and 12.7 to $25 \mathrm{~mm} / \mathrm{s}$, respectively.

Figures 3(a) and (b) show that the strain parameter, $\varepsilon^{*}$, rises with increase in laser power and decrease in scanning speed. The peak temperature during AM increases with increasing laser power and decreasing scanning speed. The ratio of laser power to scanning speed is the heat input per unit length and higher heat input results in a larger melt pool and greater distortion on solidification. The higher peak temperature, in turn, enhances the thermal strain (equation 1). The peak temperature also rises as the deposition moves to the upper layers away from the substrate and the rate of heat loss through the substrate reduces [16]. Hence, the thermal strain increases during the deposition of upper layers as indicated in Figure 3(c). However, depending on the deposition conditions, the build may attain a steady state after many layers of deposition and the thermal strain may become almost constant, particularly for longer track lengths. For example, the thermal strain in a multi-layer build of SS 316 becomes almost constant after fifteen layers as shown in Figure 3(c). The number of layers to attain a steady state when no further appreciable increase in thermal strain takes place depends on process parameters, build geometry and alloy properties. General Electric makes jet engine fuel nozzles in Auburn, Alabama in 20 
micrometer thick layers and deposits thousands of layers at very high scanning speeds without any measurable distortion. [17] This practice is consistent with our results that lower heat input is effective in mitigating distortion.

Figure 3 further shows that the thermal strain is relatively higher for IN 718 and Ti-6Al$4 \mathrm{~V}$ deposits in comparison with that for SS 316 for a given processing condition. In order to understand the relative magnitudes of thermal strain for the alloys considered, their thermophysical properties need to be considered. The Ti-6Al-4V melt pool is usually larger than that for the other alloys considered due to its lower density. Consequently, pronounced shrinkage of the pool and strain occurs in Ti-6Al-4V parts. Likewise, the deposits of IN 718 encounter high thermal strains due to its high coefficient of volume contraction. For a given set of processing conditions, IN 718 parts form large melt pools because of its low melting temperature. Shrinkage of large melt pools results in pronounced thermal strain.

Figure 3 shows that in addition to laser power and scanning speed other factors such as the alloy properties, the number of deposited layers and processing conditions affect thermal strain significantly. In order to understand these effects, the thermal strain parameter is correlated with three important non-dimensional numbers, Fourier number, Marangoni number and the non-dimensional temperature. The Fourier number is the ratio of heat dissipation rate to heat storage rate. Marangoni number represents the strength of the convective transport of heat in melt pool and the non-dimensional temperature is an indicator of the extent of overheating of the melt pool [18].

Reduction of thermal strain and distortion requires efficient dissipation of heat to avoid localized accumulation of heat. A non-dimensional parameter that embodies both heat diffusion and accumulation of heat is the Fourier number $(F)$ :

$$
F=\alpha \tau / w^{2}
$$

where $\alpha, \tau$ and $w$ refer to thermal diffusivity, characteristic time scale and length, respectively. The characteristic time can be expressed as $L / V$, where, $L$ and $V$ are the pool length and scanning speed respectively. Length of the molten pool is calculated using the heat transfer and fluid flow model as shown in Figure 1. So, equation 2 can be re-written as:

$$
F=\alpha / V L
$$

Both rapid heat dissipation and reduction of heat accumulation result in lower peak temperature, higher Fourier number and smaller molten pool length. Therefore higher Fourier number results 
in lower thermal strain and distortion. In contrast, rigidity of the substrate can counter thermal distortion in AM [5] as shown in equation (1). Figure 4(a) shows the variation of the strain parameter, $\varepsilon^{*}$ as function of Fourier number and rigidity of the substrate for three different heat inputs. The thermal strain parameter reduces with increase in both the Fourier number and the rigidity, EI, of the structure. For a set of process variables, the effects of Fourier number and rigidity of the structure on thermal strain follow nearly the same trend for all three alloys, which is expected. The figure shows that the thermal strain increases with heat input. This behavior is attributed to more pronounced heat accumulation and decrease in Fourier number when the heat input is increased. Since decrease in laser power and smaller layer heights can reduce the rate of heat accumulation and thus, increase the Fourier number, they are practical means to control the thermal distortion in AM $[3,4,19]$.

Convection is the primary mechanism of heat transfer in the molten pool during AM [35]. The convective transport of heat within the molten pool is driven primarily by the spatial variation of interfacial tension, also referred to as the Marangoni stress [20]. The shape and size of the molten pool is affected by the magnitude of the convective velocity of liquid metal that is expressed by the Marangoni number, $M a$ :

$$
M a=-\frac{d \gamma}{d T} \frac{L \Delta T}{\eta \alpha}
$$

where $\eta$ is the viscosity, $\alpha$ is the thermal diffusivity of the alloy, $L$ is the characteristic length of the molten pool, which is taken as the width of the molten pool, $\Delta T$ is the difference between the maximum temperature inside the pool and the solidus temperature of an alloy, and $\frac{d \gamma}{d T}$ is the sensitivity of surface tension with respect to temperature. For most alloys without any surface active elements, this quantity is negative. The overall molten pool volume is also affected significantly by the density of an alloy powder for a particular heat input. Lighter alloys tend to form larger molten pools and undergo greater volume contraction and thermal distortion. Therefore a combined effect of density of alloy powder and Marangoni number on the thermal strain parameter is examined.

Figure $4 \mathrm{~b}$ shows an increase in thermal strain with rise in Marangoni number and decrease in density of alloy powder for a particular set of process variables. The effect of Marangoni number and powder density follows a nearly similar trend for all the three alloys 
considered here for a particular processing condition. Increase in heat input leads to both higher peak temperature and Marangoni number, and higher thermal strain. Thus, reducing heat input by selecting either a lower laser power or a higher scanning speed or both, when possible, is a practical method to reduce thermal strain.

Equation (1) shows that the strain parameter, $\varepsilon^{*}$, increases with rise in peak temperature and $\Delta T$. In particular, higher peak temperature, $T_{P}$, results in greater volume shrinkage during solidification. A non-dimensional temperature $T^{*}$ can reveal the effect of process variables on thermal strain due to rise in peak temperature:

$$
T^{*}=\frac{T_{P}-T_{A}}{T_{L}-T_{A}}
$$

where, where $T_{A}$ and $T_{L}$ are the ambient temperature and liquidus temperature, respectively. The term $\beta T^{*}$ is a measure of the volumetric contraction. Since a lighter alloy and a larger liquid pool is more susceptible to thermal distortion, a combined effect of $\beta T^{*}$, pool length and alloy density on strain parameter is examined in Figure 4c. It is clear that the thermal strain increases with $\beta T^{*}$ and pool length and decreases with density, as expected. Low heat input and rapid heat dissipation can effectively reduce the peak temperature and pool dimension and reduce strain. The effect of the individual alloy properties such as viscosity and density at very high heat input and peak temperature appears to exhibit alloy specific trend as observed in Figs 4(b) and (c). However, both 4(b) and 4(c), clearly show how the strain parameter is affected by Marangoni number and non-dimensional temperature.

Apart from distortion, process variables also affect microstructure and mechanical properties. For example, in 316 stainless steel parts made by AM, formation of detrimental $\delta$ ferrite can be avoided and superior mechanical properties can be achieved by selecting low heat inputs [21-23]. It is also established that low heat input (100-500 W and $2000 \mathrm{~mm} / \mathrm{s})$ suppresses formation of intermetallic compounds and provides good mechanical properties of some nickel base super-alloy builds [24,25]. Facchini et al. [26] reported martensite formation and limited ductility in additively manufactured Ti-6Al-4V parts for a wide range of processing conditions. However, $\mathrm{Xu}$ et al. [27] showed that martensite formation can be avoided and fine lamellar alpha and beta structure can be achieved by careful adjustment of focal offset ( 0 to $2 \mathrm{~mm}$ ), travel speed $(1029 \mathrm{~mm} / \mathrm{s})$ and laser power $(375 \mathrm{~W})$. Therefore, it would be important to examine the impact 
of process variables on distortion, microstructure and properties to achieve defect free, structurally sound and reliable components fabricated by AM.

In summary, the results presented here provide, for the first time, a quantitative basis for minimizing thermal distortion in AM based on scientific principles and important nondimensional numbers. It is found that low heat input sufficient for maintaining adequate interlayer bonding is beneficial for the control of thermal strain. A decrease in laser power and layer height can also result in higher Fourier number and lower thermal strain. A reduction in laser power and an increase in scanning speed can reduce Marangoni number and nondimensional temperature and decrease thermal strain. Likewise, alloys with lower heat capacity and higher thermal diffusivity will be susceptible to higher peak temperature, larger pool volume and higher thermal strain. Apart from careful selection of the process parameters to mitigate distortion, microstructural characterization and evaluation of mechanical properties of the components should be undertaken to produce high quality parts.

\section{Acknowledgement}

We acknowledge the support from US Department of Energy NEUP Grant DE-NE0008280. One of the author (T.M.) also acknowledges the support from American Welding Society research fellowship grant 179466.

\section{References}

[1] I. Gibson, D.W. Rosen, B. Stucker, Additive manufacturing technologies, Springer, New York, 2010.

[2] D.D. Gu, W. Meiners, K. Wissenbach, R. Poprawe, Int. Mater. Rev. 57 (2012) 133.

[3] V. Manvatkar, A. De, T. DebRoy, Mater. Sci. Technol. 31 (2015) 924.

[4] V. Manvatkar, A. De, T. DebRoy, J. Appl. Phys. 116 (2014) 124905.

[5] T. Mukherjee, J.S. Zuback, A. De, T. DebRoy, Sci. Rep. 6 (2016) 19717. (Available at: http://www.nature.com/articles/srep19717 )

[6] T. Mukherjee, J.S. Zuback, A. De, T. DebRoy, 7th International Symposium on HighTemperature Metallurgical Processing, TMS, Nashville, TN, (2016) 469.

[7] J.C. Heigel, P. Michaleris, T.A. Palmer, J. Mater. Process. Technol. 220 (2015) 135.

[8] E.R. Denlinger, J.C. Heigel, P. Michaleris, T.A. Palmer, J. Mater. Process. Technol. 215 (2015) 123.

[9] P. Mercelis, J.P. Kruth, Rapid Prototyping J. 12 (2006) 254. 
[10] A. De, T. DebRoy, Sci. Technol. Weld Join 16 (2011) 204.

[11] C. Li, C.H. Fu, Y.B. Guo, F.Z. Fang. J. Mater. Process. Technol. 229 (2016) 703.

[12] L.M. Sochalski-Kolbus, E.A. Payzant, P.A. Cornwell, T.R. Watkins, S.S. Babu, R.R. Dehoff, M. Lorenz, O. Ovchinnikova, C. Duty. Metal. Mater. Trans. A 46 (2015) 1419.

[13] A.S. Wu, D.W. Brown, M. Kumar, G.F. Gallegos, W.E. King. Metal. Mater. Trans. A 45, (2014) 6260.

[14] K.C. Mills, Recommended values of thermo-physical properties for selected commercial alloys, Woodhead Publishing, Cambridge, 2002.

[15] V.D. Manvatkar, A.A. Gokhale, G.J. Reddy, A. Venkataramana, A. De. Metall. Mater. Trans. A 42 (2011) 4080.

[16] H.L. Wei, J. Mazumder, T. DebRoy, Sci. Rep. 5 (2015) 16446. (Available at: http://www.nature.com/articles/srep16446)

[17] M. LaMonica, GE, the world's largest manufacturer, is on the verge of using 3-D printing to make jet parts, MIT Technology Review. (Available at: http:// www.technologyreview.com/s/513716/additive-manufacturing ).

[18] V.E. Maarten, F.A. Bender, J.P. Kruth, Rapid Prototyping J. 14 (2008) 15.

[19] J. Mazumder, A. Schifferer, J. Choi, Mater. Res. Innov. 3 (1999) 118.

[20] T. DebRoy, S.A. David, Rev. Mod. Phys. 67 (1995) 85.

[21] M.H. Farshidianfar, A. Khajepour, A.P. Gerlich, J Mater. Process. Technol. 231 (2016) 468.

[22] K. Zhang, S. Wang, W. Liu, X. Shang, Mater. \& Design, 55 (2014) 104.

[23] A. Yadollahi, N. Shamsaei, S.M. Thompson, D.W. Seely, Mater. Sci. Eng. A, 644 (2015) 171.

[24] P.M. Kenney, D.E. Lindley, General Electric Company, 2013, U.S. Patent Application 14/440, 154.

[25] H. Qi, M. Azer, A. Ritter, Metal. Mater. Trans. A, 40 (2009) 2410.

[26] L. Facchini, E. Magalini, P. Robotti, A. Molinari, S. Höges, K. Wissenbach, Rapid Prototyping J. 16 (2010) 450.

[27] W. Xu, M. Brandt, S. Sun, J. Elambasseril, Q. Liu, K. Latham, K. Xia, M. Qian, Acta Mater. 85 (2015) 74. 
Table 1. Thermo-physical properties of SS 316, Ti-6Al-4V and IN 718 [14]

\begin{tabular}{|c|c|c|c|}
\hline Properties & SS 316 & Ti-6Al-4V & IN 718 \\
\hline Liquidus temperature (K) & 1733 & 1928 & 1609 \\
\hline Solidus temperature (K) & 1693 & 1878 & 1533 \\
\hline Thermal conductivity & $11.82+0.0106 \mathrm{~T}$ & $1.57+1.6 \times 10^{-2} \mathrm{~T}-$ & $0.56+2.9 \times 10^{-2} \mathrm{~T}-$ \\
$(\mathrm{W} / \mathrm{m} \mathrm{K})$ & $330.9+0.563 \mathrm{~T}-$ & $492.4+0.025 \mathrm{~T}-$ & $36.04+0.026 \mathrm{~T}-4$ \\
& $4.015 \times 10^{-4} \mathrm{~T}^{2}+$ & $4.18 \times 10^{-6} \mathrm{~T}^{2}$ & $\times 10^{-6} \mathrm{~T}^{2}$ \\
\hline Specific heat (J/ kg K) & $9.465 \times 10^{-8} \mathrm{~T}^{3}$ & & 8100 \\
\hline Density (kg/m $\left.{ }^{3}\right)$ & 7800 & 4000 & $4.8 \times 10^{-5}$ \\
\hline $\begin{array}{c}\text { Volumetric expansion co- } \\
\text { efficient (/K) }\end{array}$ & $5.85 \times 10^{-5}$ & $2.5 \times 10^{-5}$ & $5 \times 10^{-3}$ \\
\hline Viscosity (kg/m s) & $7 \times 10^{-3}$ & $4 \times 10^{-3}$ & 1.82 \\
\hline Co-efficient of surface & 1.5 & 1.52 & \\
\hline tension (N/m) & & & \\
\hline
\end{tabular}

Table 2. Process parameters used for calculations [15]

\begin{tabular}{cccccc}
\hline $\begin{array}{c}\text { Laser } \\
\text { power }(\mathrm{W})\end{array}$ & $\begin{array}{c}\text { Beam radius } \\
(\mathrm{mm})\end{array}$ & $\begin{array}{c}\text { Scanning } \\
\text { speed } \\
(\mathrm{mm} / \mathrm{s})\end{array}$ & $\begin{array}{c}\text { Layer } \\
\text { thickness } \\
(\mathrm{mm})\end{array}$ & $\begin{array}{c}\text { Substrate } \\
\text { thickness }(\mathrm{mm})\end{array}$ & $\begin{array}{c}\text { Powder flow } \\
\text { rate }(\mathrm{gm} / \mathrm{s})\end{array}$ \\
\hline $190-270$ & 0.5 & $12.7-25$ & 0.38 & 4 & 0.416 \\
\hline
\end{tabular}



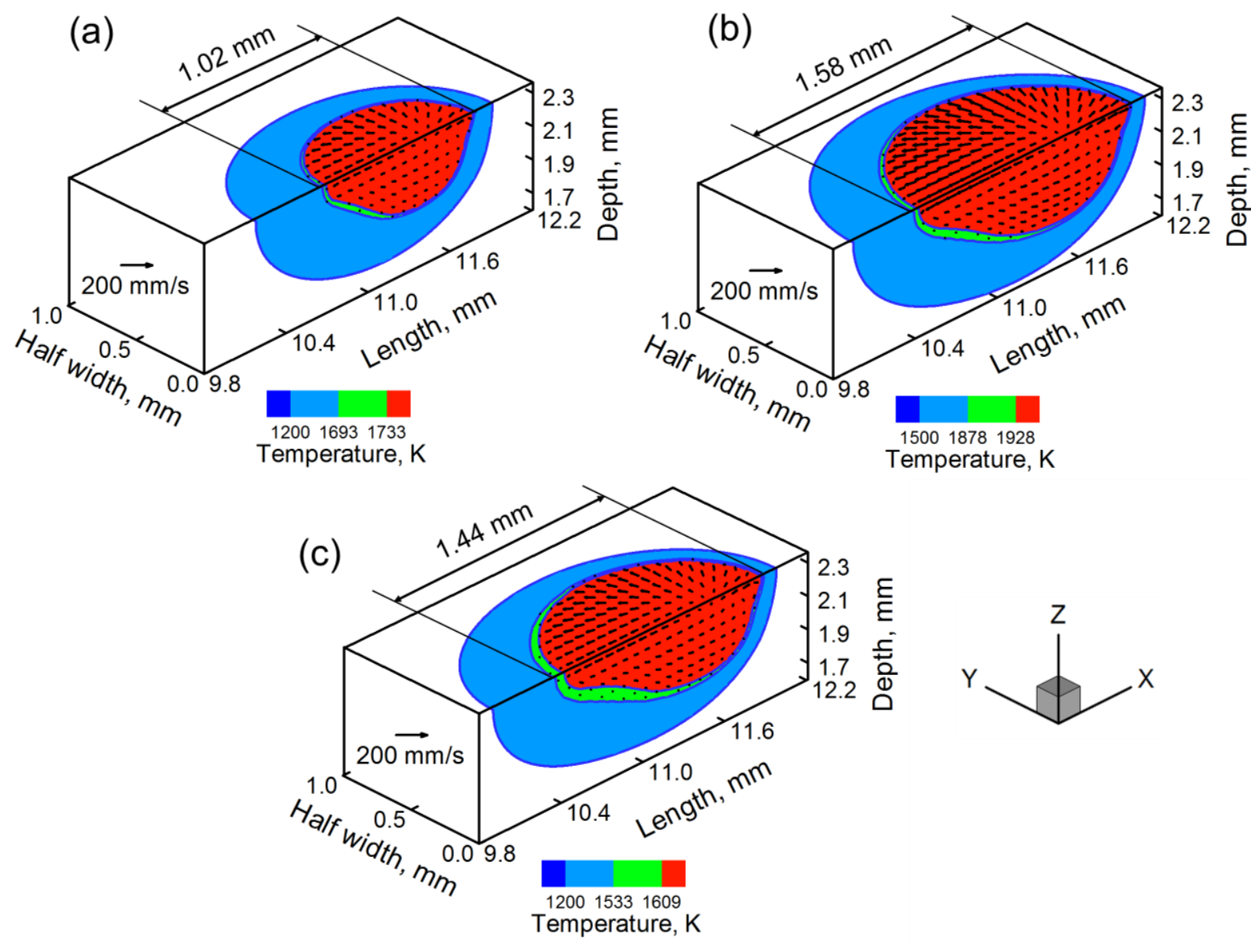

Figure 1. Computed temperature and velocity fields at the mid length during the deposition of the $2^{\text {nd }}$ layer of (a) SS 316 (b) Ti-6Al-4V and (c) IN 718. These results are for $210 \mathrm{~W}$ laser power and $12.7 \mathrm{~mm} / \mathrm{s}$ scanning speed. Scanning direction is along the positive $\mathrm{x}$-axis. 


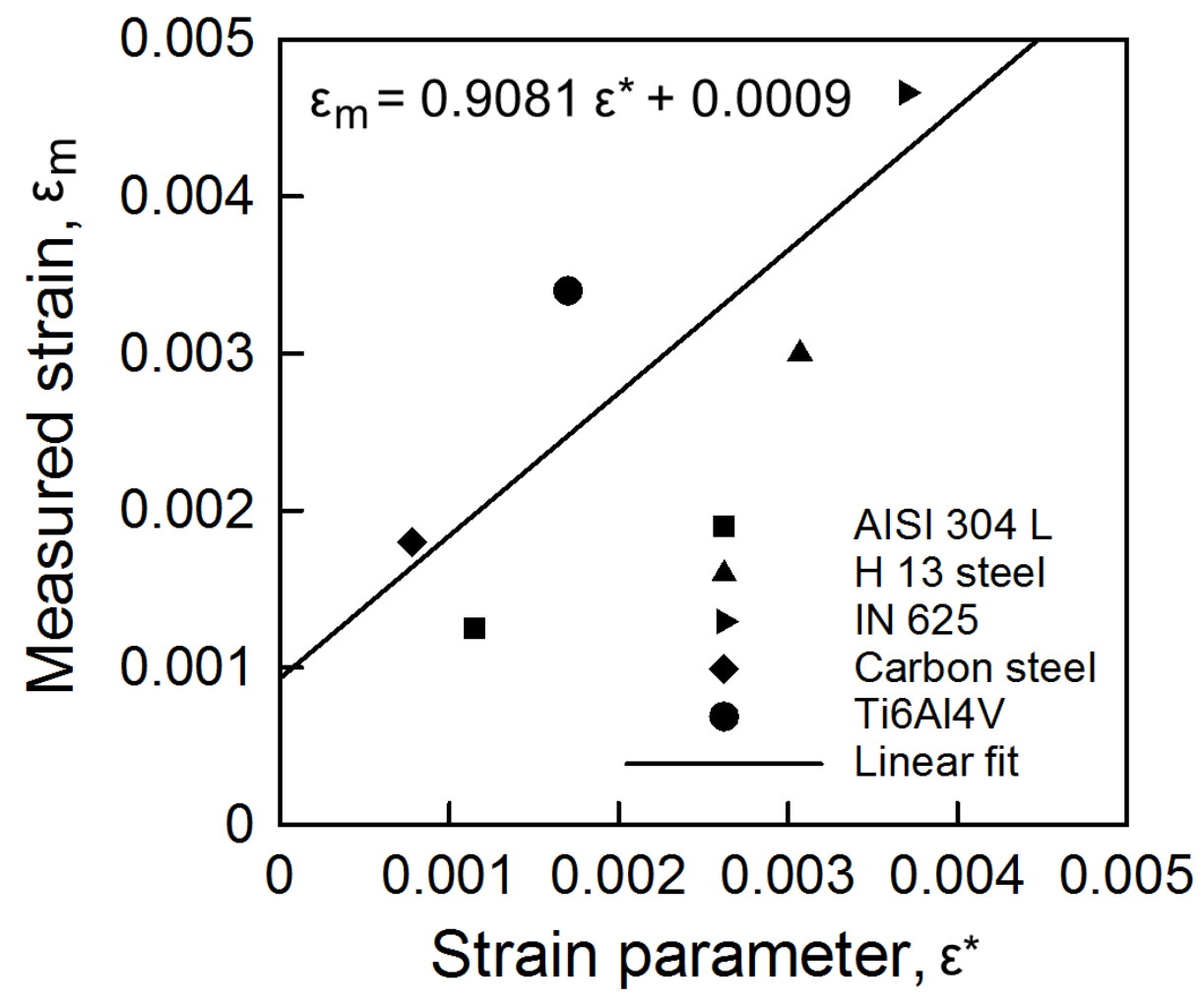

Figure 2. Linear correlation between the experimentally measured thermal strain during deposition and the non-dimensional thermal strain for five alloys [5] 

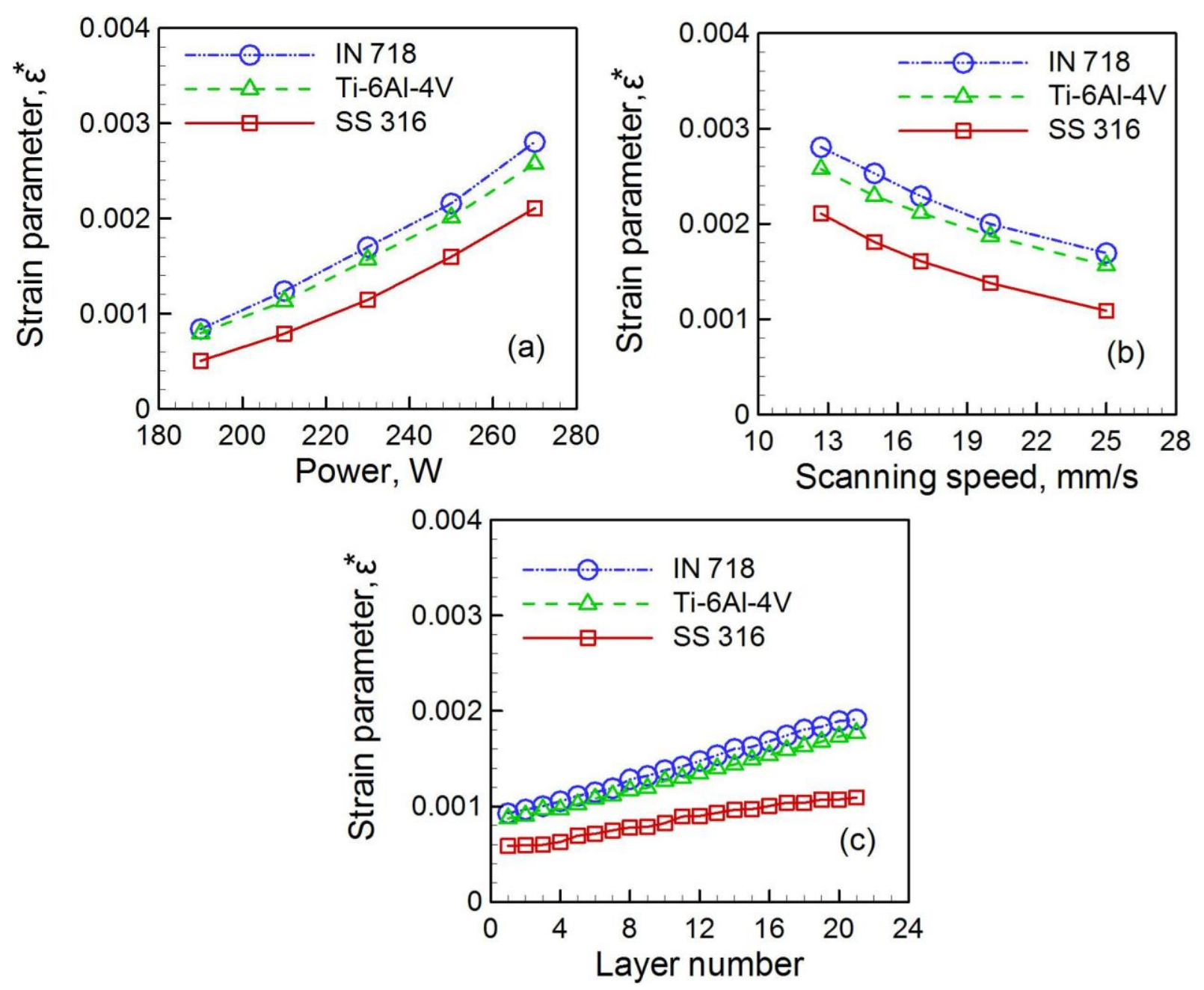

Figure 3. Variation of non-dimensional strain as function of (a) laser power for a constant scanning speed of $12.7 \mathrm{~mm} / \mathrm{s}$ and (b) scanning speed for a constant laser power of $270 \mathrm{~W}$ (c) layer number for a constant laser power of $230 \mathrm{~W}$ and scanning speed of $20 \mathrm{~mm} / \mathrm{s}$ 

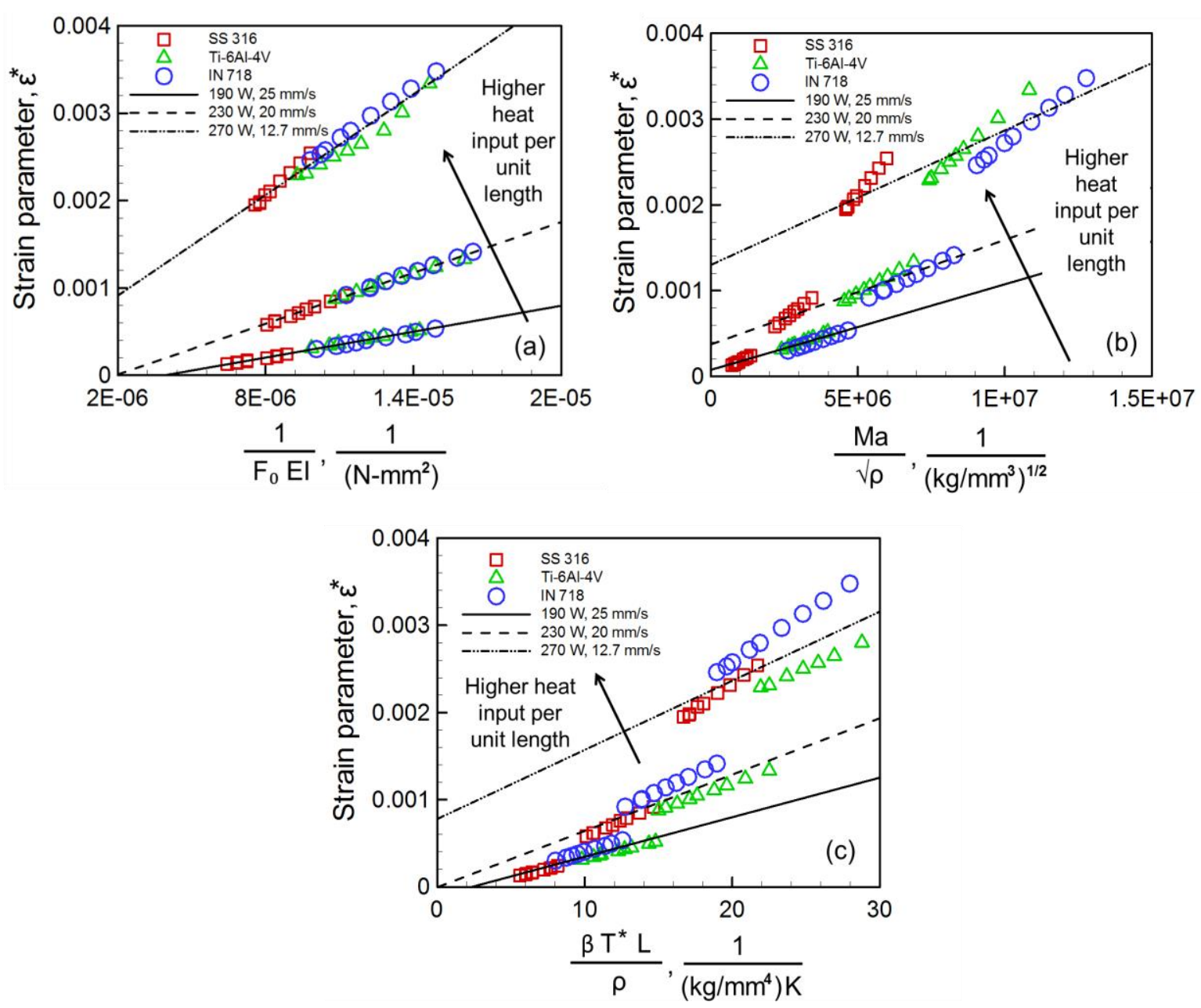

Figure 4. Variation of non-dimensional strain as function of (a) Fourier number (b) Marangoni number, and (c) non-dimensional temperature, for three heat inputs per unit length 


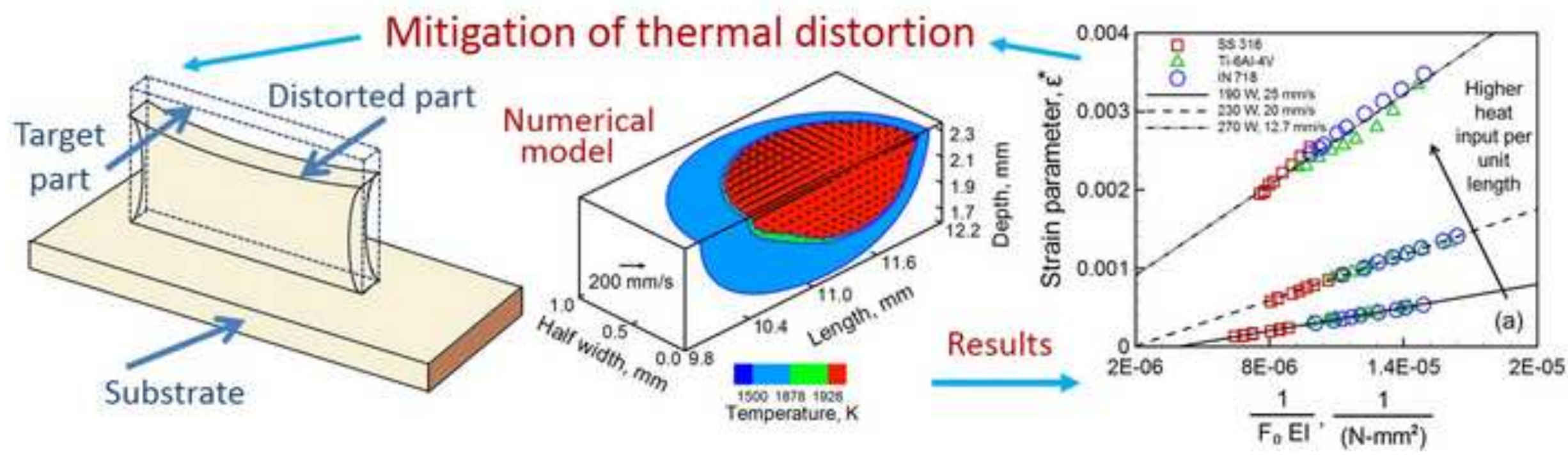

